\title{
Induction of Accelerated Atherosclerosis in Mice: The "Wire-Injury" Model
}

\author{
Adelina Curaj ${ }^{1}$, Wu Zhoujun $^{1}$, Mareike Staudt ${ }^{1}$, Elisa A. Liehn ${ }^{1,2}$ \\ ${ }^{1}$ Institute for Molecular Cardiovascular Research, RWTH Aachen University ${ }^{2}$ Human Genetic Laboratory, University of Medicine and Pharmacy
}

\section{Corresponding Author}

Elisa A. Liehn

eliehn@ukaachen.de

\section{Citation}

Curaj, A., Zhoujun, W., Staudt, M., Liehn, E.A. Induction of Accelerated Atherosclerosis in Mice: The "WireInjury" Model. J. Vis. Exp. (162), e54571, doi:10.3791/54571 (2020).

\section{Date Published}

August 25, 2020

DOI

$10.3791 / 54571$

URL

jove.com/video/54571

\section{Abstract}

Atherosclerosis is a proliferative fibro-inflammatory disease developing in the arterial wall, inducing a deficient blood flow or a lack of blood flow. Moreover, by rupture of the defective vascular wall, atherosclerosis induces occlusive thrombus formation, which represents the main cause of myocardial infarction or stroke and the most frequent cause of death. Despite the advances in the cardiovascular field, many questions remain unanswered, and additional basic research is essential to improve our understanding of the molecular mechanisms during atherosclerosis and its effects. Due to limited clinical studies, there is a need for representative animal models recreating atherosclerotic conditions such as neointima formation after stent implantation, balloon angioplasty, or endarterectomy. Since the mouse presents many advantages and is the most frequently used model for studying molecular processes, the current study proposes an invasive procedure of endothelial denudation, also known as the wire-injury model, which is representative of the human condition of neointima formation in arteries after revascularization procedures.

\section{Introduction}

Atherosclerosis is the main pathology underlying cardiovascular events such as myocardial infarction or stroke. The main mechanisms triggering acute cardiovascular syndromes are plaque rupture, superficial erosion, and thrombus formation. There are multiple clinical situations connected to the plaque development: native atherosclerotic plaque, restenosis after endarterectomy, and restenosis after balloon angioplasty with/without stent implantation ${ }^{1}$. After arterial injury, suppression of the inflammatory processes ${ }^{2,3}$ and the recovery of the endothelial compartment are essential to prevent further complications ${ }^{1}$. Clinical research is limited to tissue and blood samples due to ethical considerations, costs, and a lack of knowledge in basic mechanisms. For these reasons, there is a need to study molecular mechanisms in animal models ${ }^{4-6}$, which can recreate the clinical conditions. Our model of accelerated neointima formation in the context of atherosclerosis is the result of many years of experience in the implementation of these models in small animals ${ }^{7-11}$. The mouse model is the most attractive model for research, due to its ease of handling, the 
ability to have large animal groups due to low costs related to animal purchase and care, and the availability of various transgenic and knockout strains.

The major disadvantage of the mouse model is the small size of the main arteries subjected to atherosclerotic disease (the carotid artery, the aorta, and the femoral artery), which requires qualified surgical expertise and skills to manipulate the vessels and to invasively induce an atherosclerotic plaque. Therefore, the model of accelerated neointima formation, in the context of restenosis after endarterectomy or stent implantation, proposed in this paper is presented with a step-by-step guideline and suggestions to ease the introduction for interested personnel. Another disadvantage is that the denudation is made on the normal arterial wall, and therefore, the neo-intima formation will be moderate compared with the clinical situation. The high level of plasma cholesterol reached in apolipoprotein $\mathrm{E}$ knockout $\left(\mathrm{Apoe}^{-/-}\right)$ mice fed with a high fat-diet creates a proper pro-inflammatory environment needed for the neo-intima formation.

The surgery is performed under a stereomicroscope. The carotid artery is exposed by a median incision in the ventral cervical area. Anatomical structures on top of and surrounding the carotid artery are minimally manipulated to reduce post-surgical inflammation. The carotid artery bifurcation is exposed. To induce accelerated neointima formation, internal and external carotid arteries are prepared for blood flow cessation and subsequent common carotid artery denudation. In conclusion, the method can be learned by personnel with minimal experience in animal surgeries.

\section{Protocol}

Experiments presented in this paper are performed according to the German law and to the European animal care guidelines. The animals are bred in the animal facility of the Institute for Laboratory Animal Science, University Hospital Aachen, Germany, under supervision of Prof. Dr. R. Tolba and Dr. A. Teubner (animal welfare officer).

\section{Animal Care}

1. Keep the mice in a specialized care unit, ensuring proper access to food and specialized veterinary control and treatment. If the animals are moved or purchased from third parties, please ensure a one week accommodation period before undergoing the procedure.

\section{Hyperlipidemia Inducement}

1. Feed 6 - 8 week old, 18 - $20 \mathrm{~g}$, female (optionally) $\mathrm{ApoE}^{-/-}$mice with an atherogenic diet $(21 \%$ fat, $0.15 \%$ cholesterol, $19.5 \%$ casein, wt/wt) one week prior to the surgical procedure and continue the diet until the atherosclerotic plaque analysis is to be performed.

\section{Surgical Preparation}

1. Anesthetize the mice using an intraperitoneal injection of $100 \mathrm{mg} / \mathrm{kg}$ ketamine by bodyweight and $10 \mathrm{mg} / \mathrm{kg}$ xylazine by bodyweight. Confirm proper anesthetization prior to surgery by the lack of reflexes and whisker movement. Place a small amount of sterile eye ointment in the eye to minimize drying.

2. Ensure the maintenance of sterile conditions to avoid infections during surgery by using sterile materials and instruments.

3. Shave the mice in the ventral neck region. Disinfect the skin with betadine prior to incision. Make a $1 \mathrm{~cm}$ skin incision in the median region of the neck area, on top of the trachea. 
4. Separate the two fat bodies to ensure a proper view over the tracheal region. Use retractors to hold the muscle layer and expose the carotid artery. If present, perform the blunt-dissection of the thin muscle layer covering the carotid artery.

5. Use sharp curved forceps to separate the carotid artery from the vagus nerve and jugular vein. Thus, the bifurcation area with the internal and external carotid artery should be visible. Use $0.9 \% \mathrm{NaCl}$ in order to avoid tissue dryness during the surgical procedure.

\section{Wire-Injury}

1. Place a $7 \mathrm{~cm}$ long $0 / 5$ silk suture under the carotid artery, proximal to the aortic arch. Make an open loop, ready to be closed at any time.

2. Place two $0 / 7$ silk sutures (each $1.5 \mathrm{~cm}$ long) around the external carotid artery: one loop close to the bifurcation point, and one loop as distal as possible. Prepare them as an open loop, ready to be closed at any time.

3. Place one $0 / 7$ silk suture $(1.5 \mathrm{~cm}$ long $)$ under the internal carotid artery. Prepare it as an open loop, ready to be closed at any time.

4. Position the mouse table with the mouse head towards the operator to ensure proper positioning for the guidewire insertion during the denudation (Figure 1A).

5. Under the microscopic view, stop the blood flow through the common carotid artery by holding and pulling the ends of the $0 / 5$ silk suture with hemostat forceps.

6. Immediately after the common carotid artery ligature, close the suture loops placed on the internal carotid artery and the distal suture on the external carotid artery tightly (Figure 1B).
7. Perform a small incision (arteriotomy, half of the vessel diameter) distal to the external carotid artery, between the two loops, using small scissors (Figure 1C). If the incision is too big, please follow the troubleshooting instructions (see the Discussion).

8. Use commercially polished guide wires or use in-house specialized personnel to polish the guide wires. Disinfect the 14 inch polished flexible guide-wire with alcohol and moisten it in a droplet of $0.9 \% \mathrm{NaCl}$ to ensure proper sliding into the vessel.

9. Insert the guide-wire into the common carotid artery via the transverse arteriotomy of the external carotid artery (Figure 1D). Obtain endothelial denudation by passing the guide wire along the vessel while rotating. Repeat this procedure three times. Maintain the same amplitude of rotational movement in each mouse to increase the reproducibility.

10. Close the proximal loop on the external carotid artery tightly. Restore the blood flow in the carotid artery by cutting the suture around common artery and the suture around the internal carotid artery.

\section{Suture and Recovery}

1. Remove the retractors and return the muscle layer and the two fat bodies to the physiological position.

2. Close the skin with three separated sutures $0 / 6$, if echocardiographic measurements are needed. If no imaging is needed, use metallic clips to close the skin.

3. Lay the mouse down on its left side under the infrared light until it wakes up. Do not leave an animal unattended nor in the company of other animals until fully recovered. 
4. For future identification, mark the mouse using the local system. Ask the animal welfare officer from the local institution.

\section{Analysis of the Atherosclerotic Plaque}

1. Anesthetize the mice at the end-time point using an intraperitoneal injection of $100 \mathrm{mg} / \mathrm{kg}$ ketamine by bodyweight and $10 \mathrm{mg} / \mathrm{kg}$ xylazine by bodyweight. Confirm proper anesthetization by the lack of reflexes and whisker movement.

2. Perform exsanguination by retro-orbital or cardiac puncture and collect the blood for further analysis ${ }^{2}$.

3. Disinfect the skin with betadine. Open the thoracic cavity and remove the right auriculum of the heart. Perfuse phosphate buffered solution through left ventricle to remove the remaining blood from the vessel and then perfuse $4 \%$ PFA to fix the tissue.

4. If no fixation is required, explant the carotid artery immediately after washing ${ }^{2,4,11}$. Perform standard protocols with analyses of interest: paraffin embedding, cryosection, mRNA or protein analysis, etc.

5. For morphometrical measurements, carefully explant the carotid artery including the bifurcation, with minimal manipulation, as proximal to the aortic arch using curved forceps and small scissors.

6. Embed the carotid artery in the paraffin block using standard embedding protocols. To perform transversal sectioning, place the carotid artery upright on bifurcation. Cut $5 \mu \mathrm{m}$ thick serial sections starting with the bifurcation and collect them all on coated histological slides (Figure 2A).
7. Stain every $10^{\text {th }}$ section using Movat staining to highlight the laminas $2,4,11$. After collecting microscopic pictures of all vessels (using a 10X objective), measure the lumen, as well as the internal and external lamina for each section, using special designed software ${ }^{2,4,11}$, as shown in Figure 2B. Calculate the intimal growth and media of the vessels.

8. Analyze smooth muscle cells and macrophage content, or endothelial recovery in serial sections, using usual immunohistological staining ${ }^{2}$ (Figure 2C).

\section{Representative Results}

The atherosclerotic plaque induction procedure takes 15 - 20 min and shows a minimal mortality rate, mostly due to the bleeding occurring during the procedure. After surgery, the mice recover from anesthesia within $20-25 \mathrm{~min}$. No physical impairment, such as paralysis, or feeding disturbance was observed after the surgery.

The wire-injury induces a de-endothelialization, mimicking vascular lesions after balloon denudation or stentimplantation. Immediately after injury, the denuded vascular wall will be covered with a layer of thrombocytes, which mediates and favors the adhesion of the monocytes ${ }^{12}$. Activated smooth muscle cells from the media will proliferate and migrate into the intimal spaces, forming the neointima. Other progenitors for smooth muscle cells will migrate from the blood (estimated to be $40 \%$ ) and contribute to the neointima growth. The plaque formation will end after the complete re-endothelialization, usually 4 weeks after the wireinjury. 
The neointima formation can be assessed using Movat staining. The plaque size is calculated for each slide using software as shown in Figure 2B. The total plaque size (left carotid artery) can vary between $70,000-100,000 \mu \mathrm{m}^{2}$, while the control vessel size (right carotid artery) can vary between $7,000-8,000 \mu m^{2}$. These values depend largely on the surgeon. Therefore, we strongly recommend using the same surgeon during the experiments for the same study.

The developed plaque resembles in stent restenosis, which predominately consists of proliferated and migrated smooth muscle cells from the media. The cellular composition determined by immunological staining procedures shows that the smooth muscle cell content is approximately $30-40 \%$, while macrophages are found in $15-25 \%$ of the neointima of the injured vessel. The re-endothelialization can be measured after staining for an endothelial marker, and calculated as the percentage of circumference stained over the entire circumference of the lumen. Usually the re-endothelialization reaches $80-90 \%$ after 3 weeks, and should almost be complete after 4 weeks (Figure 2C). To track the plaque growth during its development, the same analysis can be repeated for every time-point after the wire-injury, depending on the interest and the subject studied (see Table 1).

A

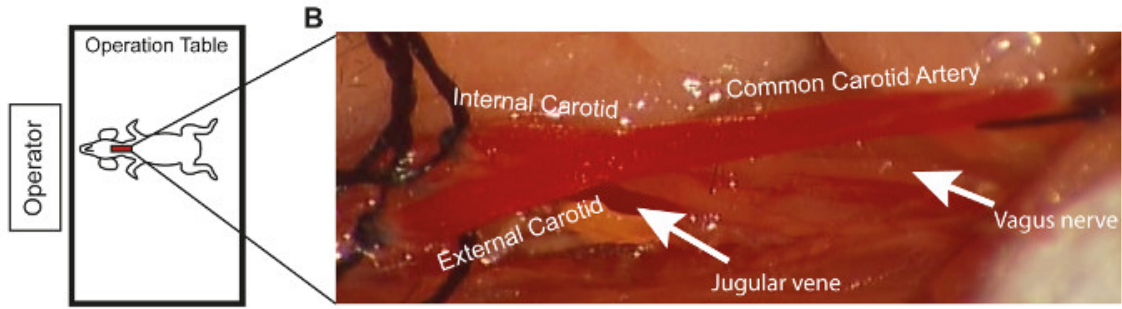

C

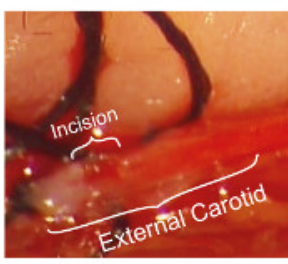

D

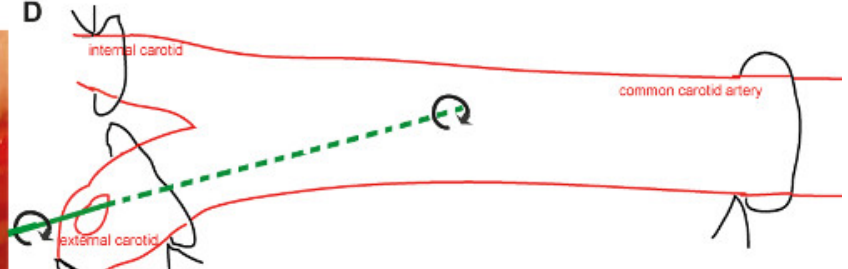

Figure 1. Schematic Representation of Operative Procedure. (A) The positioning of the operation table toward the operator during the wire-injury procedure (B) Enlarged view of the common carotid artery and its branches, as it appears under the microscope at 10X magnification (C) The size of the incision in the external carotid artery under the microscope at 10X magnification (D) Schematic representation of wire-injury procedure using the 14 inch guide wire. Please click here to view a larger version of this figure. 
A
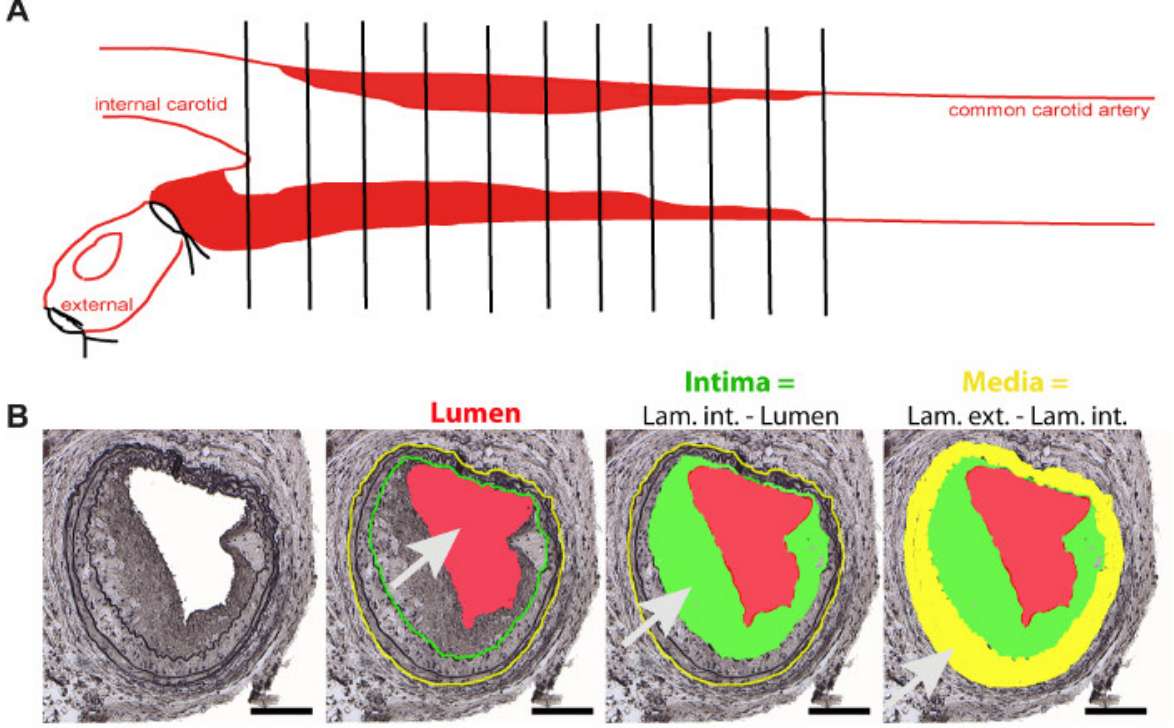

C

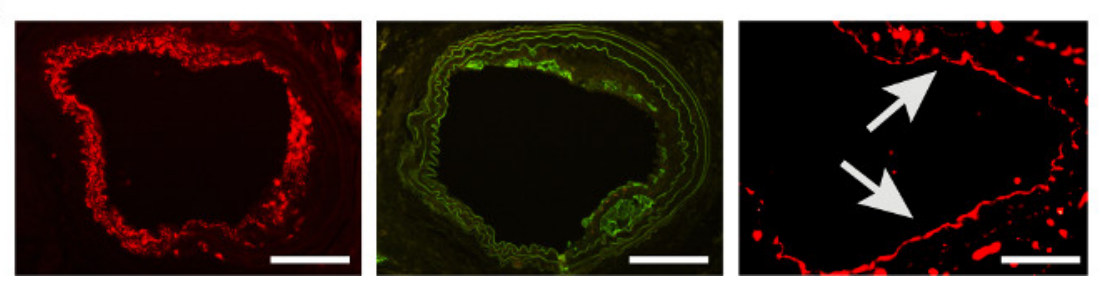

Figure 2. Analysis of Restenosis Plaque. (A) Schematic representation of plaque analysis in the common carotid artery, 4 weeks after wire-injury induction (B) Neointima formation 4 weeks after the wire-injury and schematic representation of main parameters used for analysis. Intima (green area) is the difference between the lumen (red) and the lamina interna (green line). Media (yellow area) is the difference between the lamina externa (yellow line) and interna (green line). Scale bar 100 $\mu \mathrm{m}$ (C) Representative images of the staining of the main cell types involved in neointima formation. Smooth muscle cells (smooth muscle actin -red, scale bar $100 \mu \mathrm{m}$ ), macrophages (Mac 2- green, scale bar $100 \mu \mathrm{m}$ ) and endothelial cells (CD31red, arrows, scale bar $50 \mu \mathrm{m})$. Please click here to view a larger version of this figure. 


\begin{tabular}{|c|c|c|c|c|c|}
\hline Time & Thrombus & Plaque $\left(\boldsymbol{\mu m}^{2}\right)$ & $\begin{array}{c}\text { Macrophages } \\
(\% \text { from Plaque })\end{array}$ & $\begin{array}{c}\text { Smooth } \\
\text { muscle cells } \\
(\% \text { from Plaque })\end{array}$ & $\begin{array}{c}\text { Re- } \\
\text { endothelialization } \\
(\% \text { lumen } \\
\text { circumference })\end{array}$ \\
\hline 1 day & present & 0 & 0 & 0 & 0 \\
\hline 1 week & - & $<30000$ & $>10$ & $<50$ & $<50$ \\
\hline 2 weeks & - & $<70000$ & $15-25$ & $30-40$ & $80-90$ \\
\hline 3 weeks & - & $70000-100000$ & $15-25$ & $30-40$ & complete \\
\hline 4 weeks & - & $>10$ & $<50$ & $>50$ \\
\hline
\end{tabular}

Table 1. Time-dependent Plaque's Development.

Copyright $\odot 2020$ JoVE Creative Commons Attribution-NonCommercial-NoDerivs 3.0 Unsorted

jove.com

August $2020 \cdot 162 \cdot$ e54571 Page 7 of 12

License 


\begin{tabular}{|c|c|c|c|}
\hline Model & Animals & Advantages & Disanvantages \\
\hline \multirow[t]{2}{*}{$\begin{array}{l}\text { Diet-induced native } \\
\text { atherosclerosis }\end{array}$} & Small & $\begin{array}{l}\text { - mimics the atherosclerosis } \\
\text { - pathology } \\
\text { - } \text { ease of handling } \\
\text { - no stress for the animals } \\
\text { - low costs related to animal } \\
\text { purchase and care } \\
\text { - availability of various } \\
\text { transgenic and knockout } \\
\text { strains }\end{array}$ & $\begin{array}{l}\text { - low reproductibility } \\
\text { - high variance } \\
\text { - increased animal's } \\
\text { number required } \\
\text { - increased waiting time }\end{array}$ \\
\hline & Big & $\begin{array}{l}\text { - mimics the atherosclerosis } \\
\text { pathology } \\
\text { - ease of handling } \\
\text { - no surgery } \\
\text { - no stress for the animals }\end{array}$ & $\begin{array}{l}\text { - low reproductibility } \\
\text { - high variance } \\
\text { - increased animal's } \\
\text { number required }\end{array}$ \\
\hline \multirow[t]{2}{*}{ Balloon dilatation } & Small & $\begin{array}{l}\text { - mimics restenosis after } \\
\text { balloon angioplasty } \\
\text { - low costs related to animal } \\
\text { purchase and care } \\
\text { - availability of various } \\
\text { transgenic and knockout } \\
\text { strains }\end{array}$ & $\begin{array}{l}\text { - small size of the main } \\
\text { arteries } \\
\text { - requires qualified surgical } \\
\text { expertise } \\
\text { - balloons very expensive } \\
\text { - denudation is made on the } \\
\text { normal arterial wall } \\
\text { - existence of appropriate } \\
\text { equipment } \\
\text { - risks of complications as } \\
\text { bleeding or paralysis }\end{array}$ \\
\hline & Big & $\begin{array}{l}\text { - mimics restenosis after } \\
\text { balloon angioplasty }\end{array}$ & $\begin{array}{l}\text { - denudation is made on the } \\
\text { normal arterial wall }\end{array}$ \\
\hline
\end{tabular}




\begin{tabular}{|c|c|c|c|}
\hline & & $\begin{array}{l}\text { - ease of handling } \\
\text { - use of devices for humans }\end{array}$ & \\
\hline Wire Injury & Small & $\begin{array}{l}\text { - mimics restenosis after } \\
\text { balloon angioplasty } \\
\text { - ease of handling } \\
\text { - minimal mortality rate } \\
\text { - low costs related to animal } \\
\text { purchase and care } \\
\text { - availability of various } \\
\text { transgenic and knockout } \\
\text { strains } \\
\text { - no physical impairment }\end{array}$ & $\begin{array}{l}\text { - small size of the main } \\
\text { arteries } \\
\text { - requires less qualified } \\
\text { surgical expertise } \\
\text { - denudation is made on the } \\
\text { normal arterial wall } \\
\text { - existence of appropriate } \\
\text { equipment }\end{array}$ \\
\hline \multirow[t]{2}{*}{ Stent implantation } & Small & $\begin{array}{l}\text { - mimics restenosis and } \\
\text { thrombosis after stent } \\
\text { implantation } \\
\text { - low costs related to animal } \\
\text { purchase and care } \\
\text { - availability of various } \\
\text { transgenic and knockout } \\
\text { strains }\end{array}$ & $\begin{array}{l}\text { - small size of the main } \\
\text { arteries } \\
\text { - requires qualified surgical } \\
\text { expertise } \\
\text { - small stents not available } \\
\text { - denudation is made on the } \\
\text { normal arterial wall } \\
\text { - increased mortality } \\
\text { - existence of appropriate } \\
\text { equipment } \\
\text { - risks of complications as } \\
\text { bleeding or paralysis }\end{array}$ \\
\hline & Big & $\begin{array}{l}\text { - mimics restenosis } \\
\text { and thrombosis after stent } \\
\text { implantation } \\
\text { - ease of handling }\end{array}$ & $\begin{array}{l}\text { denudation is made on the } \\
\text { normal arterial wall }\end{array}$ \\
\hline
\end{tabular}


Table 2. Advantages and Disadvantages of Existent Models of Arterial Injury.

\section{Discussion}

In this paper, we provide useful tips to perform the wireinjury procedure even by personnel with minimal experience in animal surgeries. There are two critical steps in performing this procedure: the incision of the external carotid artery and the insertion of the wire. The incision in the external carotid artery needs to be performed as far as possible from the bifurcation, in order to ensure enough remaining material (Figure 1C). The incision should not be too large, due to the risk of cutting the entire vessel. The second critical step is the high risk of bleeding during the arteriotomy and the insertion of the guide wire if blood flow is not efficiently discontinued. Moreover, endothelial denudation might not take place or arterial rupture is possible if the guide wire is not properly introduced in the lumen vessel. To avoid this, the surface of the guide wire must be carefully polished before the operation.

To optimize the protocol, the position of the operating-table with the mouse-head towards the surgeon ensures a better view, accessibility and control for the proper guide wire manipulation. Moreover, to increase the reproducibility, use the same guide wire in all of the studies. Since the wire size does not change, it is important to consider and eliminate all the possible differences between the mice by using the same gender, age and weight for all mice included in a study. Thereafter, Evans-Blue staining will help the surgeon determine the efficiency of the denudation. The existence of appropriate equipment is a prerequisite for the success of the procedure. A 10X stereomicroscope is essential for performing this procedure. The proper preparation of the guide-wire (for example polishing it) is crucial. Therefore, we strongly recommend that the guide wire preparation be performed by specialized technical personnel where available.

There are many troubleshooting steps in this protocol. If incising the external carotid artery near the bifurcation, carefully bind the externa, near the bifurcation, so no bleeding occurs. During cutting, the external carotid artery cannot be seen. Therefore, consider the bifurcation at the level of silk suture. Collect sections when the silk suture disappears. If the incision in the external carotid artery is too large and the vessel is ruptured, ensure that the blood flow into the carotid communis and internal carotid artery is effectively interrupted and try to find the opening of the vessel using forceps. After introducing the guide wire and performing the denudation, bind the vessel near the bifurcation. During cutting, start to collect when the silk from the suture starts to disappear. If arterial rupture occurs during the denudation with the guide wire, check under the microscope if the guide wire is properly polished.

Despite the similarity of the wire-injury model to clinical situations, many groups are focused on native atherosclerosis in mice, or they choose invasive atherosclerosis inductions, such as balloon angioplasty in rats or rabbits, because of the lack of trained personnel who can perform small animal surgeries. Despite the benefits of using rabbits/rats, e.g. no need for miniaturized equipment, neither rat models nor rabbit models offer a variety of different knock-out strains, in terms of studying molecular mechanisms involved in neointima growth and in-stent thrombosis. 
The existing models for studying in-stent restenosis in mice are difficult, require high surgical skills, and have high risks of complications such as bleeding or paralysis. For example, the mechanical injury or stent-implantation into the thoracic aorta via femoral artery is accompanied by a high mortality rate $(35 \%)$ due to hind leg paralysis or bleeding ${ }^{13-15}$. We also describe stent implantation in the carotid artery of a mouse $^{16}$. The procedure is similar; however, the tissue processing for analysis is complicated and is not available to all laboratories ${ }^{16}$. The carotid artery is directly accessible, not only for operation procedures, but also for existing imaging methods such as ultrasound imaging. Other injury inductions in the carotid arteries in mice can be done using electrical devices $^{17}$. This method is easy to perform and ensures high reproducibility. However, it induces injury in all vessel layers, which is not identical with mechanical injury. Balloon applications have benefits, e.g. the adjustment to the vessel diameter in line with the clinical practice and has strong influence on the pathological outcome. Even though mouse balloons are available, they are very expensive and therefore, not widely used. Instead, the wire-injury is the established method, mimicking in-stent stenosis.

The denudation is performed on the normal arterial wall, though with an atherosclerotic background. Therefore, the neointima formation will be moderate compared to the clinical situation. The high number of preclinical models demonstrates that none of the models fulfill all of the criteria necessary to uncover the entirety of the cellular and molecular mechanisms leading to the pathophysiology in humans (see Table 2).

After performing the wire-injury procedure, other biological and molecular analysis can be performed to identify cells, proteins, mRNAs, microRNAs, genes or other biomarkers, which can be used as therapeutic targets to develop new treatment strategies for atherosclerosis, and in particular for neointima formation after vascular injury. If available, the plaque growth can be monitored using high frequency ultrasound or other high-resolution imaging techniques. Moreover, mastering this technique would give the operator the opportunity to adapt the protocol to other invasive atherosclerosis inducement models, such as collar placement, partial ligation or even stent implantation.

\section{Disclosures}

There are no disclosures by the authors.

\section{Acknowledgments}

This work was supported by Interdisciplinary Centre for Clinical Research IZKF Aachen (junior research group to E.A.L.) within the faculty of Medicine at RWTH Aachen University. We also thank Mrs. Roya Soltan for help with the immunohistochemistry staining.

\section{References}

1. Simsekyilmaz, S., Liehn, E.A., Militaru, C., \& Vogt, F. Progress in interventional cardiology: challenges for the future. Thromb Haemost. 113 (3), 464-472 (2015).

2. Kubo, N., McCurdy, S., \& Boisvert, W.A. Defective Fas Expression on Bone Marrow Derived Cells Alters Atherosclerotic Plaque Morphology in Hyperlipidemic Mice. Discoveries. 3 (1), e37 (2015).

3. Saffarzadeh, M., et al. Characterization of rapid neutrophil extracellular trap formation and its cooperation with phagocytosis in human neutrophils. Discoveries. 2 (2), e19 (2014). 
4. Lindner, V., Fingerle, J., \& Reidy, M.A. Mouse model of arterial injury. Circ Res. 73 (5), 792-796 (1993).

5. Schwartz, R.S., et al. Preclinical evaluation of drug-eluting stents for peripheral applications: recommendations from an expert consensus group. Circulation. 110 (16), 2498-2505 (2004).

6. Schwartz, R.S., et al. Restenosis and the proportional neointimal response to coronary artery injury: results in a porcine model. J Am Coll Cardiol. 19 (2), 267-274 (1992).

7. Curaj, A., et al. Noninvasive molecular ultrasound monitoring of vessel healing after intravascular surgical procedures in a preclinical setup. Arterioscler Thromb Vasc Biol. 35 (6), 1366-1373 (2015).

8. Liehn, E.A., Schober, A., \& Weber, C. Blockade of keratinocyte-derived chemokine inhibits endothelial recovery and enhances plaque formation after arterial injury in ApoE-deficient mice. Arterioscler Thromb Vasc Biol. 24 (10), 1891-1896 (2004).

9. Liehn, E.A., Zernecke, A., Postea, O., \& Weber, C. Chemokines: inflammatory mediators of atherosclerosis. Arch Physiol Biochem. 112 (4-5), 229-238 (2006).

10. Simsekyilmaz, S., et al. Role of extracellular RNA in atherosclerotic plaque formation in mice. Circulation. 129 (5), 598-606 (2014).

11. Wu, Z., et al. Rhodamine-loaded intercellular adhesion molecule-1-targeted microbubbles for dual-modality imaging under controlled shear stresses. Circ Cardiovasc Imaging. 6 (6), 974-981 (2013).

12. Schober, A., et al. Crucial role of the CCL2/CCR2 axis in neointimal hyperplasia after arterial injury in hyperlipidemic mice involves early monocyte recruitment and CCL2 presentation on platelets. Circ Res. 95 (11), 1125-1133 (2004).

13. Ali, Z.A., et al. Increased in-stent stenosis in ApoE knockout mice: insights from a novel mouse model of balloon angioplasty and stenting. Arterioscler Thromb Vasc Biol. 27 (4), 833-840 (2007).

14. Chamberlain, J., et al. A novel mouse model of in situ stenting. Cardiovasc Res. 85, 38-44 (2010).

15. Rodriguez-Menocal, L., et al. A novel mouse model of in-stent restenosis. Atherosclerosis. 209 (2), 359-366 (2010).

16. Simsekyilmaz, S., et al. A murine model of stent implantation in the carotid artery for the study of restenosis. J Vis Exp. e50233, (2013).

17. Schroder, K., et al. NADPH oxidase Nox2 is required for hypoxia-induced mobilization of endothelial progenitor cells. Circ Res. 105 (6), 537-544 (2009). 